\section{Management and outcome of sight- threatening diabetic retinopathy in pregnancy}

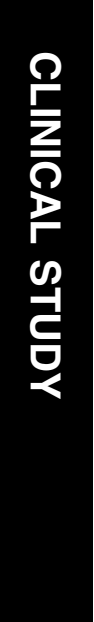

${ }^{1}$ Eye \& Ear Clinic

Royal Victoria Hospital

Belfast, Northern Ireland, UK

${ }^{2}$ Queen's University of Belfast Northern Ireland, UK

${ }^{3}$ Regional Centre for Endocrinology and Diabetes Royal Victoria Hospital Belfast

Northern Ireland, UK

Correspondence: WC Chan MRCOphth

Eye \& Ear Clinic

Royal Victoria Hospital

Grosvenor Road

Belfast BT12 6BA

Northern Ireland, UK

Tel: + 442890240503

Fax: + 442890330744

E-mail: wingchan365@

hotmail.com

Received: 25 June 2003 Accepted in revised form: 1 September 2003

Published online:

20 February 2004

This paper was presented as a poster at the 2003 Oxford Ophthalmological Congress, Oxford.

None of the authors has a financial or proprietary interest in any material mentioned.
Abstract

Aims To report the management and outcomes of sight-threatening diabetic retinopathy in pregnancy.

Methods A retrospective review of 8 diabetic females who developed pregnancy related sight-threatening diabetic retinopathy requiring treatment over a 12-year period. Results In total, 16 eyes of eight patients were included in this series. The mean age of the patients at presentation was 30.75 years $\pm 3.8 \mathrm{SD}$ and the mean duration of diabetes was 21.0 years \pm 5.1 SD. The mean follow-up period was 46.75 months \pm 47.2 SD. A total of $87.5 \%$ of patients showed progression of diabetic retinopathy during pregnancy, $71 \%$ of which were in the sight-threatening proliferative category. In the postpartum period, $81 \%$ of patients continued to progress to proliferative diabetic retinopathy, requiring panretinal photocoagulation and multiple other surgical procedures. In all, $69 \%$ of eyes retained visual acuity equal to or better than $0.3 \log$ MAR units (6/12).

Conclusion Sight-threatening diabetic retinopathy in pregnancy is a rare disease, but it can have devastating consequences for mother and child. Laser photocoagulation should be considered for pregnant women with severe preproliferative diabetic retinopathy. Proliferative diabetic retinopathy may not regress postpartum. Close followup should be extended in the postpartum period in this group of patients until the retinopathy is stabilised. The presence of combined rhegmatogenous and tractional retinal detachment and neovascular glaucoma were associated with the worst outcome. Eye (2004) 18, 826-832. doi:10.1038/sj.eye.6701340 Published online 20 February 2004

Keywords: diabetes mellitus; diabetic retinopathy; pregnancy
WC Chan'1, LT Lim², MJ Quinn', FA Knox', D McCance ${ }^{3}$ and RM Best ${ }^{1}$

\section{Introduction}

Diabetic retinopathy is one of the major causes of preventable blindness in the UK and the USA in those aged between 24 and 64 years. ${ }^{1}$ For diabetic women, this may coincide with fertility and childbearing years, and the implications for a blind mother, to take care of and bring up children, are significant. It is well known that diabetic retinopathy may worsen during pregnancy $^{2-4}$ and that its severity may regress in the postpartum period. ${ }^{5,6}$ However, the exact mechanism responsible for progression of diabetic retinopathy during pregnancy is still not entirely clear. Factors thought to be of significance include poor metabolic control, ${ }^{7}$ long duration of diabetes, ${ }^{8,9}$ severity of retinopathy at baseline, ${ }^{10}$ rapid normalisation of glycaemic control, ${ }^{11,12}$ hypertension, ${ }^{4,13}$ and changes in retinal blood flow. ${ }^{14}$ A study by Phelps et $\mathrm{al}^{7}$ and the Diabetes in Early Pregnancy Study (DIEP) ${ }^{10}$ found that patients in whom retinopathy was most likely to progress had both the poorest control at baseline and the largest improvement during early pregnancy. DIEP also found that duration of diabetes of more than 15 years, and severity of existing retinopathy were most important factors in the development of and progression to severe retinopathy in pregnancy. Rosenn et al ${ }^{13}$ found that patients with chronic hypertension or pregnancy-induced hypertension or both had a higher rate of progression of retinopathy. Increased retinal blood flow, as a consequence of hyperdynamic circulatory state in pregnancy, may induce endothelial damage at the capillary level, which may be the responsible factor. ${ }^{14}$ The aims of this case series were two-fold. Firstly, we report the characteristics of eight patients whose diabetic retinopathy worsened during pregnancy and whose retinopathy did not regress postpartum. Secondly, we report on the management and outcome of these difficult cases. 


\section{Materials and method}

This was a retrospective review of eight patients who developed sight-threatening diabetic retinopathy during pregnancy and who did not show postpartum regression over a 12 year period from 1990 to 2002. These patients were recruited from a population of pregnant diabetic women who attended the combined Antenatal Diabetic Clinic at the Royal Jubilee Maternity Hospital, Belfast, Northern Ireland and the specialist Diabetic Eye Clinic at the Royal Victoria Hospital, Belfast, Northern Ireland. During the period between 1990 and 2002, there were 540 pregnant diabetic mothers who attended these two tertiary specialist clinics, and their hospital records were scrutinised. Sight-threatening diabetic retinopathy was defined as diabetic retinopathy with features associated with a particular high risk of severe visual loss as defined in the Diabetic Retinopathy Study (DRS). ${ }^{15}$

In the Diabetic Eye Clinic, all patients underwent clinical examination including distance Snellen visual acuity, anterior segment examination, and retinal examination. Follow-up visits were carried out every 4 weeks or sooner if considered to be necessary.

Fluorescein angiography was arranged only if judged to be of clinical benefit. In the combined Antenatal Diabetic Clinic, all patients underwent measurement of blood pressure, serum blood glucose, and HbA1c level.

Follow-up visits were carried out every 2 weeks until 32 weeks gestation, and then weekly until delivery.

Information was obtained from the medical records of the patients. Data abstracted included the age of the patient, duration of diabetes, smoking status, parity, serum glucose level, blood pressure, $\mathrm{HbA1c}$, gestational age at delivery, outcome of pregnancy, Snellen visual acuity at presentation, status of diabetic retinopathy at each follow-up, time and type of eye treatment, final visual outcome, and total duration of follow-up. Diabetic retinopathy was classified into background diabetic retinopathy (BGDR), preproliferative diabetic retinopathy (PPDR), proliferative diabetic retinopathy (PDR), that is, neovascularisation at the disc (NVD) or neovascularisation elsewhere (NVE) subjectively quantified into number of disc areas and its complications including vitreous haemorrhage (VH), tractional retinal detachment (TRD), and neovascular glaucoma (NG).

\section{Results}

\section{Demographic details}

The mean age was 30.75 years $\pm 3.8 \mathrm{SD}$ and the mean duration of diabetes was 21.0 years $\pm 5.1 \mathrm{SD}$. The mean duration of follow-up was 46.7 months \pm 47.2 SD. Two of eight patients were smokers.

\section{Medical details}

Mean serum blood glucose was $8.7 \mathrm{mmol} / 1 . \pm 4.1 \mathrm{SD}$ for trimester $1,7.7 \mathrm{mmol} / 1 \pm 1.9 \mathrm{SD}$ for trimester 2 , and $7.7 \mathrm{mmol} / \mathrm{l} \pm 1.6 \mathrm{SD}$ for trimester 3 . Mean $\mathrm{HbA1c}$ was $9.1 \% \pm 3.8 \mathrm{SD}$ for trimester $1,7.1 \% \pm 1.5 \mathrm{SD}$ for trimester 2 , and $8.3 \% \pm 3.0 \mathrm{SD}$ for trimester 3 . The values of $\mathrm{HbA} 1 \mathrm{c}$ during pregnancy are outlined in Table 1. Mean blood pressure was $130 / 78 \mathrm{mmHg} \pm 11 / 6 \mathrm{SD}$ for trimester 1 , $128 / 78 \mathrm{mmHg} \pm 10 / 6 \mathrm{SD}$ for trimester 2 , and $128 /$ $78 \mathrm{mmHg} \pm 8 / 5 \mathrm{SD}$ for trimester 3 .

\section{Parity and pregnancy outcome}

Seven out of the eight patients in this cohort had more than one pregnancy when the progression of diabetic retinopathy was noted during this study period. All the patients in this cohort had normal blood pressure throughout the pregnancy and had normal pregnancy outcomes.

\section{Visual acuity on presentation (Table 2)}

On presentation, $94 \%$ of eyes had visual acuity equal to or better than 0.3 . $\log$ MAR units $(6 / 12)$ and one eye had a visual acuity of $1.3 \log$ MAR units $(3 / 60)$ due to combined tractional and rhegmatogenous retinal detachment.

\section{Fundus features on presentation (Table 3)}

Three eyes had previous pan retinal photocoagulation (PRP). Eight eyes had BGDR, five eyes had PPDR, and one eye had combined TRD (when first seen in the first trimester).

\section{Follow-up findings during pregnancy (Table 3)}

In all, 14 eyes showed signs of progression of diabetic retinopathy during pregnancy. Four eyes progressed from BGDR to PPDR and 10 eyes progressed to PDR one eye occurred in the first trimester, four eyes occurred

Table 1 Values of HbA1c during pregnancy

\begin{tabular}{lccc}
\hline Patient no. & $\begin{array}{c}\text { HbA1c } \\
\text { trimester 1 (\%) }\end{array}$ & $\begin{array}{c}\text { HbAlc } \\
\text { trimester 2 (\%) }\end{array}$ & $\begin{array}{c}\text { HbA1c } \\
\text { trimester 3 (\%) }\end{array}$ \\
\hline 1 & 9.4 & 5.8 & 6.0 \\
2 & 9.2 & 8.9 & 9.1 \\
3 & 17.7 & 15.9 & 15.2 \\
4 & 8.1 & 8.9 & 7.9 \\
5 & 8.1 & 7.5 & 7.7 \\
6 & 8.5 & 8.2 & 8.1 \\
7 & 6.2 & 6.3 & 6.8 \\
8 & 5.4 & 5.1 & 5.4 \\
\hline
\end{tabular}


Table 2 Visual results

\begin{tabular}{llccr}
\hline $\begin{array}{l}\text { Patient } \\
\text { no. }\end{array}$ & Eye & $\begin{array}{l}\text { Initial } \\
\text { VA:Snellen } \\
\text { (logMAR) }\end{array}$ & Final VA & $\begin{array}{r}\text { Change } \\
\text { of lines }\end{array}$ \\
\hline 1 & & $6 / 6(0.0)$ & $6 / 9(0.2)$ & -2 \\
& $\mathrm{R}$ & $6 / 6(0.0)$ & $6 / 9(0.2)$ & -2 \\
2 & $\mathrm{~L}$ & $6 / 9(0.2)$ & $\mathrm{NPL}$ & -14 \\
& $\mathrm{R}$ & $6 / 12(0.3)$ & $\mathrm{NPL}$ & -13 \\
3 & $\mathrm{~L}$ & $6 / 7.5(0.1)$ & $6 / 12(0.3)$ & -2 \\
& $\mathrm{R}$ & $6 / 7.5(0.1)$ & $6 / 7.5(0.1)$ & 0 \\
4 & $\mathrm{~L}$ & $6 / 6(0.0)$ & $6 / 60(1.0)$ & -10 \\
& $\mathrm{R}$ & $6 / 6(0.0)$ & $\mathrm{NPL}$ & -16 \\
5 & $\mathrm{~L}$ & $6 / 6(0.0)$ & $6 / 6(0.0)$ & 0 \\
& $\mathrm{R}$ & $6 / 6(0.0)$ & $6 / 12(0.3)$ & -3 \\
6 & $\mathrm{~L}$ & $6 / 6(0.0)$ & $6 / 9(0.2)$ & -2 \\
& $\mathrm{R}$ & $3 / 60(1.3)$ & $6 / 24(0.6)$ & +7 \\
7 & $\mathrm{~L}$ & $6 / 6(0.0)$ & $6 / 7.5(0.1)$ & -1 \\
& $\mathrm{R}$ & $6 / 6(0.0)$ & $6 / 12(0.3)$ & -3 \\
8 & $\mathrm{~L}$ & $6 / 6(0.0)$ & $6 / 9(0.2)$ & -2 \\
& $\mathrm{R}$ & $6 / 9(0.2)$ & $6 / 12(0.3)$ & -1 \\
\hline
\end{tabular}

$\mathrm{R}=$ right; $\mathrm{L}=$ left; $\mathrm{VA}=$ visual acuity; $\mathrm{NPL}=$ no perception of light.

in the second trimester, and five eyes occurred in the third trimester. Of the 10 eyes that progressed to PDR during pregnancy, one eye developed TRD during the first trimester requiring surgery during pregnancy (Patient No. 6). Two eyes remained unchanged at PPDR during pregnancy.

\section{Intervention during pregnancy (Table 3)}

Five eyes received PRP, one eye received PRP and pars plana vitrectomy (for TRD), and 10 eyes did not receive treatment.

\section{Post. partum follow up findings (Table 3)}

A total of, 15 eyes did not show signs of regression postpartum. In all, 13 eyes progressed to PDR in the postpartum period. Seven eyes developed $\mathrm{VH}$, three eyes developed TRD, Two eyes developed NG, and two eyes developed diabetic maculopathy.

\section{Postpartum intervention (Table 3)}

All eyes that did not show signs of regression required treatment. In total, 13 eyes required further PRP and two eyes required focal laser alone. Two eyes required one pars plana vitrectomy for nonclearing $\mathrm{VH}$. Three eyes developed combined rhegmatogenous and TRD in the post partum period after initial pars plana vitrectomy for nonclearing VH (Patient Nos. 4 and 5, where Patient No. 4 required three vitrectomies, scleral buckling procedure and lensectomy in one eye and three vitrectomies, lensectomy, silicone oil and scleral buckling in the other eye, which was left aphakic and the silicone oil is still in situ with no plan for removal at present). One patient (Patient No. 2) required trabeculectomy with Mitomycin $\mathrm{C}$ and diode laser cycloablation in one eye and trabeculectomy with Mitomycin C, laser cycloablation, Ahmed valve implantation, three vitrectomies and lensectomy in the other eye for resistant NG and nonclearing $\mathrm{VH}$.

\section{Visual outcome at final visit (Table 2)}

The mean follow-up period of these patients was 46.8 . months $\pm 47.2 \mathrm{SD}$. In total, $69 \%$ of eyes retained visual acuity equal to or better than $0.3 \log$ MAR units $(6 / 12)$. One eye had a visual acuity of $0.6 \log$ MAR units $(6 / 24)$, One eye had a visual acuity of $1.0 \log$ MAR units (6/60), and three eyes were totally blind. Only two eyes had no change or improvement of visual acuity, and the remaining 14 eyes $(87.5 \%)$ had a decrease of at least 1 $\log$ MAR unit of vision $(25 \%$ had a decrease of more than $10 \log$ MAR units of vision). Despite the relatively good final visual outcome, $37.5 \%$ of eyes lost at least three lines of visual acuity.

\section{Reasons for poor visual outcome}

The worst outcomes occurred in two patients. Of the four eyes, final vision was $1.0 \log$ MAR units $(6 / 60)$ or worse at the last follow-up. The reasons for poor outcome were combined rhegmatogenous and TRD in two eyes of one patient. Surgery was successful in one eye (visual acuity of $1.0 \log$ MAR units or 6/60) but not in the other. The other patient developed end-stage NG in both eyes, with final visual acuity of no perception of light. Both patients were registered blind. Of the four eyes that developed TRD, three eyes had a final visual acuity of less than 0.3 $\log$ MAR units or $6 / 12$.

\section{Selected case reports}

\section{Case 1 (Patient 5 in Tables 1-3)}

A 36-year-old lady, with a 27-year history of type I diabetes mellitus, became pregnant for the first time. She was monitored throughout her pregnancy and her diabetic retinopathy progressed gradually from BGDR in the first and second trimester to PPDR in the third trimester of the pregnancy. Visual acuity remained 6/6 in both eyes throughout the pregnancy. At 4 weeks postpartum, she developed VH in the right eye and PRP was commenced in this eye. The left eye showed no signs of proliferative disease at that stage. After 2 weeks, neovascularisation was detected in the inferior retina in 
Table 3 Fundal findings, changes, and treatment

\begin{tabular}{|c|c|c|c|c|c|c|}
\hline $\begin{array}{l}\text { Patient } \\
\text { no. }\end{array}$ & Eye & $\begin{array}{l}\text { Fundal } \\
\text { changes at } \\
\text { presentation }\end{array}$ & $\begin{array}{c}\text { Progression } \\
\text { during pregnancy }\end{array}$ & $\begin{array}{l}\text { Treatment } \\
\text { during } \\
\text { pregnancy }\end{array}$ & $\begin{array}{l}\text { Fundal changes } \\
\text { postpartum }\end{array}$ & $\begin{array}{l}\text { Treatment } \\
\text { postpartum }\end{array}$ \\
\hline \multirow[t]{2}{*}{1} & $\mathrm{R}$ & BGDR & Yes, to PDR & PRP & PDR & PRP \\
\hline & $\mathrm{L}$ & BGDR & Yes, to PDR & PRP & PDR & PRP \\
\hline \multirow[t]{2}{*}{2} & $\mathrm{R}$ & BGDR & Yes, to PPDR & No & PDR, VH, NG & $\begin{array}{l}\text { PRP, Trab + MitoC, } \\
\text { Cyclodiode }\end{array}$ \\
\hline & $\mathrm{L}$ & BGDR & Yes, to PPDR & No & PDR, VH, NG & $\begin{array}{l}\text { PRP, Cyclodiode, } \\
\text { Trab + MitoC, } \\
\text { Ahmed Valve Implant, } \\
\text { lensectomy, PPV } \times 3\end{array}$ \\
\hline \multirow[t]{2}{*}{3} & $\mathrm{R}$ & PPDR & No, remained PPDR & No & PDR & PRP \\
\hline & $\mathrm{L}$ & PPDR & No, remained PPDR & No & PDR & PRP \\
\hline \multirow[t]{2}{*}{4} & $\mathrm{R}$ & PPDR & Yes, to PDR & No & PDR, VH, TRD & $\begin{array}{l}\mathrm{PRP}, \mathrm{SBP}, \mathrm{PPV} \times 3 \text {, } \\
\text { lensectomy }\end{array}$ \\
\hline & $\mathrm{L}$ & PPDR & Yes, to PDR & No & PDR, VH, TRD & $\begin{array}{l}\mathrm{PRP}, \mathrm{PPV} \times 3, \mathrm{SiO}, \\
\mathrm{SBP} \text {, lensectomy }\end{array}$ \\
\hline \multirow[t]{2}{*}{5} & $\mathrm{R}$ & BGDR & Yes, to PPDR & No & PDR, VH & PRP, PPV \\
\hline & $\mathrm{L}$ & BGDR & Yes, to PPDR & No & PDR, VH, TRD & $\mathrm{PRP}, \mathrm{PPV} \times 2, \mathrm{SBP}$ \\
\hline \multirow[t]{2}{*}{6} & $\mathrm{R}$ & $\begin{array}{l}\text { Inactive DR } \\
\text { (treated) }\end{array}$ & Yes, to PDR & No & PDR, VH & PRP, PPV \\
\hline & $\mathrm{L}$ & $\begin{array}{l}\text { Inactive DR } \\
\text { (treated) }\end{array}$ & Yes, to PDR and TRD & PRP, SBP, PPV & Inactive DR & No \\
\hline \multirow[t]{2}{*}{7} & $\mathrm{R}$ & PPDR & Yes, to PDR & PRP & Diabetic maculopathy & Focal laser \\
\hline & $\mathrm{L}$ & $\begin{array}{l}\text { Inactive DR } \\
\text { (treated) }\end{array}$ & Yes, to PDR & PRP & Diabetic maculopathy & Focal laser \\
\hline \multirow[t]{2}{*}{8} & $\mathrm{R}$ & BGDR & Yes, to PDR & No & PDR & PRP \\
\hline & $\mathrm{L}$ & BGDR & Yes, to PDR & No & PDR & PRP \\
\hline
\end{tabular}

$\mathrm{DR}=$ diabetic retinopathy; $\mathrm{BGDR}=$ background diabetic retinopathy; $\mathrm{PPDR}=$ preproliferative diabetic retinopathy; $\mathrm{PDR}=$ proliferative diabetic retinopathy; $\mathrm{PRP}=$ pan retinal photocoagulation; $\mathrm{PPV}=$ pars plana vitrectomy; $\mathrm{SiO}=$ silicone oil; $\mathrm{Trab}=$ Trabeculectomy; Mito $\mathrm{C}=$ mitomycin $\mathrm{C}$; $\mathrm{VH}=$ vitreous haemorrhage; $\mathrm{TRD}=$ tractional retinal detachment; $\mathrm{NG}=$ neovascular glaucoma; $\mathrm{SBP}=$ scleral buckling procedure; $\mathrm{RD}=$ retinal detachment; $\mathrm{R}=$ right; $\mathrm{L}=$ left.

the left eye and PRP was commenced in this eye. Despite treatment, both eyes continued to develop recurrent $\mathrm{VH}$, reducing vision to perception of light in both eyes 4 months postpartum. The right eye received a total of 1628 spots of laser burns and the left eye received 1795 spots of laser burns, and the application of laser was somewhat hampered by the VH. The VH failed to clear and the patient underwent left pars plana vitrectomy and indirect laser photocoagulation. At 2 weeks postsurgery, the left eye developed an inferior combined

rhegmatogenous and TRD with a tear identified at about the 4 o'clock position. The patient underwent repeat pars plana vitrectomy, epiretinal membrane removal, indirect laser photocoagulation, and scleral buckling with a No. 42 silicone encircling band. The vitreous cavity was filled with $14 \% \mathrm{C}_{3} \mathrm{~F}_{8}$. The retina remained attached. The right $\mathrm{VH}$ did not clear and this eye underwent pars plana vitrectomy and indirect laser photocoagulation with no complications. At 10 months after the first vitrectomy, the retina remained attached with a corrected vision of $6 / 6$ in the right eye and 6/12 in the left eye.

\section{Case 2 (Patient 2 in Tables 1-3)}

A 31-year-old-lady, with a 17-year history of type I diabetes mellitus, became pregnant for the second time. Her diabetic retinopathy progressed from BGDR during the first and second trimester to PPDR during the third trimester. At 3 weeks postpartum, the patient presented with a painful right eye. The right eye had developed NG and $\mathrm{VH}$ with corneal oedema, extensive rubeosis iridis, and the intraocular pressure (IOP) was $50 \mathrm{mmHg}$. The patient was treated with Diamox SR $250 \mathrm{mg}$ bd, Gt Iopidine tds, Gt Betoptic 0.5\% bd, Gt Trusopt tds, and Gt 
Minims Dexamethasone $0.1 \%$ hourly. The right eye was painful and the IOP failed to settle. Laser photocoagulation was difficult due to poor corneal view. At that stage, the left eye developed mild rubeosis iridis and $\mathrm{VH}$, and this eye was treated intensively with PRP. At 8 weeks postpartum, the right eye underwent trabeculectomy with Mitomycin C for uncontrolled NG. There was still no view for PRP. This controlled the IOP for 3 weeks, after which the IOP was raised to $52 \mathrm{mmHg}$ and the bleb failed. IOP was uncontrolled with maximal medical therapy and diode laser cycloablation was carried out. This failed to control the IOP and the right eye progressed rapidly to no perception of light in 2 weeks. In the meantime, the left eye continued to receive PRP, but the onset of VH made the application of retinal laser difficult. NG developed and the IOP was raised at $55 \mathrm{mmHg}$ despite maximal medical therapy. Urgent left trabeculectomy with Mitomycin C was carried out. This controlled the IOP for 4 weeks, until the IOP was again raised at $50 \mathrm{mmHg}$. Diode laser cycloablation was carried out but this failed to control the IOP. The patient underwent urgent Ahmed valve implant the following week, which controlled the IOP. The cornea oedema resolved but the dense $\mathrm{VH}$ failed to resolve and the vision was counting fingers in this eye. Left pars plana vitrectomy and indirect laser photocoagulation were carried out. This was complicated by recurrent VH. Repeat pars plana vitrectomy and indirect laser photocoagulation were carried out to wash out the nonclearing VH. The IOP remained controlled but the anterior and posterior proliferative disease failed to regress. Persistent nonclearing recurrent VH complicated by cataract formation required a third operation, that is, combined phacoemulsification cataract extraction, pars plana vitrectomy, and indirect laser photocoagulation. However, aggressive inflammatory response developed in the anterior chamber and the eye gradually became hypotonous and resulted in phthisis bulbi. Vision was no perception of light in both eyes 1 year postpartum.

\section{Discussion}

Rodman $e a^{3}$ reported that $8 \%$ of 201 pregnant diabetic women with no or mild BGDR at the onset of pregnancy showed progression of the diabetic retinopathy during pregnancy, and in 127 women with proliferative disease, $25 \%$ showed progression during pregnancy. In another study by Laatikainen $e t a l^{16}$ who studied 73 consecutive pregnant diabetic women, no significant progression of retinopathy was noted in patients who lacked or had minimal retinopathy at the beginning of pregnancy, but $65 \%$ of patients with frank retinopathy in the first trimester were observed to progress during pregnancy.
It is commonly believed that diabetic retinopathy regresses in the postpartum period. ${ }^{5,6}$ However, the rate and timing of regression of retinopathy postpartum in diabetic pregnant women is unknown. In this study, $87.5 \%$ of patients showed progression of retinopathy during pregnancy and $71 \%$ of these patients were in the proliferative retinopathy category. In this subgroup of patients who developed proliferative retinopathy, only $50 \%$ of these patients received treatment during pregnancy and the other $50 \%$ did not, as it was believed that regression would occur after delivery. In the postpartum period, $81 \%$ of patients continued to progress to PDR, all occurring within an 8-week period postpartum. In this study, only one eye that had treatment during pregnancy showed regression postpartum and the rest did not. These eyes required aggressive treatment postpartum. In 50\% of these patients, laser photocoagulation was hampered by the onset of $\mathrm{VH}$. The management of this subgroup of patients became especially difficult with the onset of NG and TRD. The outcome in this subgroup of patients was very poor. It is possible that the outcome in these patients would have been better if laser photocoagulation were performed earlier rather than anticipating regression after delivery. One striking feature of this cohort of patients was the degree of symmetry both in the development of diabetic retinopathy and the final outcome, and, as such, the changes in the first eye can be used as a predictor for the progression of retinopathy in the second eye.

The management of diabetic retinopathy in these difficult cases can be problematic. There is little information in the literature regarding the surgical management of diabetic retinopathy associated with pregnancy and its outcome. Regarding the surgical management, the most common indications for surgery are nonclearing VH, TRD, and NG. One patient in this cohort required pars plana vitrectomy and scleral buckling for tractional macular detachment during pregnancy (Patient No. 6). This was associated with difficulties as the surgery was technically difficult and had to be done under local anaesthesia. There was also the potential problem of a pregnant woman lying on her back for a considerable period of time. Pars plana vitrectomy is effective in clearing the $\mathrm{VH}$ and allows retinal laser treatment to be carried out. Sometimes repeated surgery is required for recurrent $\mathrm{VH}$ (Patient No. 2) or complicated by tractional or rhegmatogenous retinal detachment (Patient Nos. 4 and 5). The combination of NG and VH (Patient No. 2) was associated with the worst outcome in this study. In our experience, when surgery is required in an eye with active proliferative disease without significant prior PRP treatment, the final results were poor (Patient Nos. 2 and 4). 
The visual outcome with aggressive laser treatment was reasonable with $69 \%$ of eyes achieving a visual acuity of $6 / 12$ or better. However, $37.5 \%$ of eyes had lost at least three lines of vision despite treatment. In the subgroup with poor visual outcome, the delay in initiation of PRP was a possible factor responsible for this poor outcome.

Laser photocoagulation remains the mainstay of treatment in this condition with reasonable outcomes. ${ }^{17,18}$ Patient Nos. 2 and 5 developed PPDR during pregnancy and both patients required extensive vitreo-retinal surgery with three out of the four eyes ending up totally blind. In addition, Patient No. 4 developed proliferative changes during pregnancy requiring vitreo-retinal surgery. This would suggest that laser photocoagulation applied at the proliferative stage may be too late. In the absence of any other effective treatment modality and the potential serious consequences, we recommend that laser photocoagulation be considered at the severe preproliferative stage and it may be unwise to wait for the development of even early proliferative changes. In addition, in view of the uncertainty of the rate of regression postpartum, close follow-up is recommended after delivery.

There are several limitations in the present study, notably the weakness of an uncontrolled retrospective study and the small sample size. Only eight patients out of 540 developed sight-threatening diabetic retinopathy, thus confirming the rarity of this condition.

Some measures can be taken to try and prevent the development of sight-threatening diabetic retinopathy in pregnancy. Treatment of PDR with laser photocoagulation may lessen the progression during pregnancy. It is also important that diabetic women who may become pregnant establish good glucose control before conception. ${ }^{12}$ The value of prepregnancy counselling regarding the potential risks and consequences to vision should not be overlooked and greater patient education would be beneficial.

In summary, sight-threatening diabetic retinopathy associated with pregnancy is rare, but it can have dire consequences for mothers and their children. Therefore, we recommend that pregnant patients who exhibit progression of diabetic retinopathy to severe preproliferative changes be considered for laser photocoagulation and it may be unwise to wait for the emergence of proliferative changes, as the consequences of withholding treatment in this subgroup of patients can be devastating. The reason for recommending treatment at the severe preproliferative stage is that follow-up of such patients in the late stages of the pregnancy can be difficult especially if there are coexisting problems with their pregnancy like pre-eclampsia, and even one missed clinic attendance could be critical in such patients. In addition, PDR may not regress in the postpartum period and close follow-up should be extended in the postpartum period in this subgroup of patients until retinopathy is stabilised. In the subgroup of patients requiring surgery, the presence of combined rhegmatogenous and TRD and NG were associated with the worst outcome.

\section{References}

1 Kohner EM, Porta M. Protocols for screening and treatment of diabetic retinopathy in Europe. Eur J Ophthalmol 1991; 1: $45-54$.

2 The Diabetes Control, Complications Trial Research Group. Effect of pregnancy on microvascular complications in the Diabetes Control and Complications Trial. Diabetes Care 2000; 23: 1084-1091.

3 Rodman H, Singerman LJ, Aiello L, Merkatz IR. Diabetic retinopathy and its relationship to pregnancy. In: Merkaty T, Adams P (eds). The Diabetic Pregnancy: A Perinatal Perspectiv, Grune \& Stratton: New York 1979.

4 Klein BE, Moss SE, Klein R. Effect of pregnancy on progression of diabetic retinopathy. Diabetes Care 1990; 13: 34-40.

5 Sunness JS. The pregnant women's eye. Surv Ophthalmol 1988; 32: 219-238.

6 Serup L. Influence of pregnancy on diabetic retinopathy. Acta Endocrinol (Copenh) 1986; 22(Suppl): 122-124.

7 Phelps RL, Sakol P, Metzger BE, Jampol LM, Frienkel N. Changes in diabetic retinopathy during pregnancy. Correlations with regulation of hyperglycaemia. Arch Ophthalmol 1986; 104: 1806-1810.

8 Dibble CM, Kochenour NK, Worley RJ, Tyler FH, Swartz M. Effect of pregnancy on diabetic retinopathy. Obstet Gynecol 1982; 59: 699-704.

9 Maloney JB, Drury MI. The effect of pregnancy on the natural course of diabetic retinopathy. Am J Ophthalmol 1982; 93: 745-756.

10 Diabetes in Early Pregnancy Study. Metabolic control and progression of retinopathy. Diabetes Care 1995; 18: 631-637.

11 Laatikainen L, Teramo K, Heita-Heikurainen H, Koivisto V, Pelkonen R. A controlled study of the influence of continuous subcutaneous insulin infusion treatment on diabetic retinopathy during pregnancy. Acta Med Scand 1987; 221: 367-376

12 Chew EY, Mills JL, Metzger BE, Remaley NA, JovanovichPeterson L, Knopp RH. Metabolic control and the progression of retinopathy. The diabetes in Early Pregnancy Study. Diabetes Care 1995; 18: 631-637.

13 Rosenn B, Miodovnik M, Kranias G, Khoury J, Combs CA, Mimouni $\mathrm{F}$ et al. Progression of diabetic retinopathy in pregnancy: association with hypertension in pregnancy. Am J Obstet Gynecol 1992; 166: 1214-1218.

14 Best RM, Chakravarthy U. Diabetic retinopathy in pregnancy. Br J Ophthalmol 1997; 81: 249-251.

15 Diabetic Retinopathy Study Research Group. Four risk factors for severe visual loss in diabetic retinopathy: the third report from the Diabetic Retinopathy Study. Arch Ophthalmol 1979; 97: 654-655. 
16 Laatikainen L, Larinkari J, Teramo K. Occurrence and prognostic significance of retinopathy in diabetic pregnancy. Metab Paediatr Ophthalmol 1980; 4: 191-195.

17 Hercules BL, Wozencroft M, Gayed II, Jeacock J. Peripheral retinal ablation in the treatment of proliferative diabetic retinopathy during pregnancy. Br J Ophthalmol 1980; 64: 87-93.

18 Price JH, Hadden DR, Archer DB, Harley JM. Diabetic retinopathy in pregnancy. Br J Obstet Gynaecol 1984; 91: 11-17. 